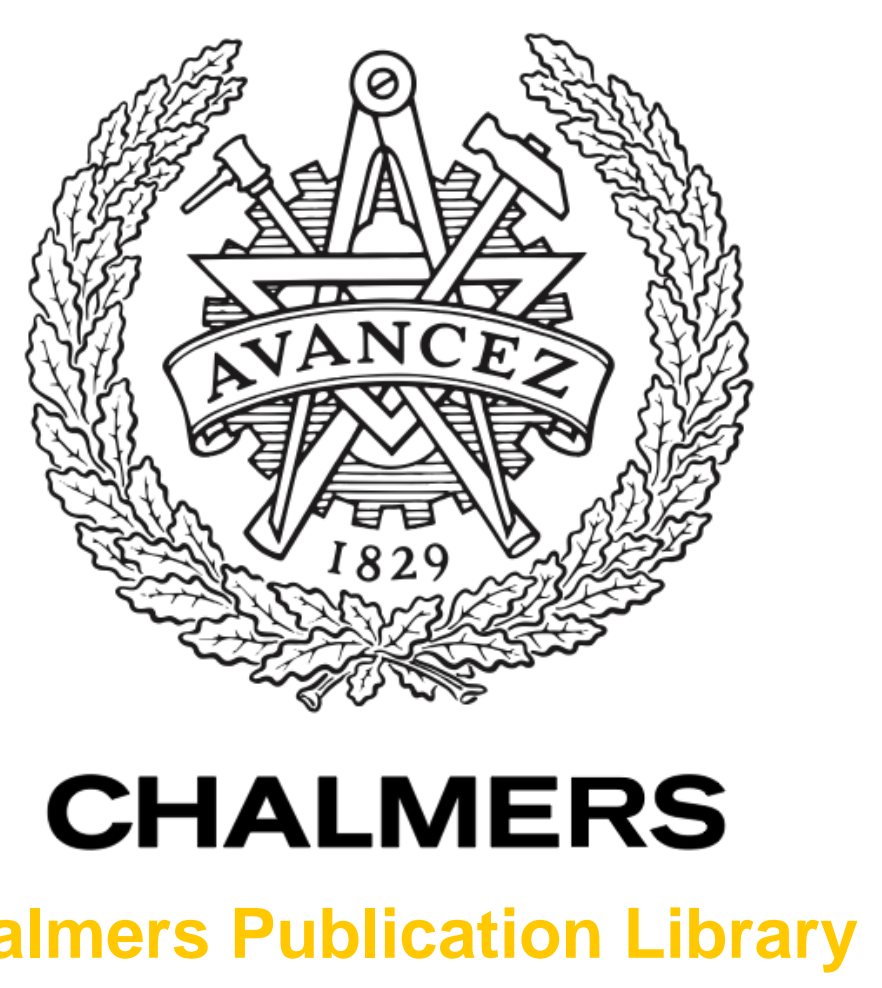

Chalmers Publication Library

\author{
Non-invasive EEG source localization using particle swarm optimization: A clinical \\ experiment
}

This document has been downloaded from Chalmers Publication Library $(\mathrm{CPL})$. It is the author's version of a work that was accepted for publication in:

34th Annual International Conference of the IEEE Engineering in Medicine and Biology Society, EMBS 2012, San Diego, CA, 28 August - 1 September 2012 (ISSN: 1557-170X)

Citation for the published paper:

Shirvany, Y. ; Edelvik, F. ; Jakobsson, S. (2012) "Non-invasive EEG source localization using particle swarm optimization: A clinical experiment". 34th Annual International Conference of the IEEE Engineering in Medicine and Biology Society, EMBS 2012, San Diego, CA, 28 August - 1 September 2012 pp. 6232-6235.

http://dx.doi.org/10.1109/EMBC.2012.6347418

Downloaded from: http://publications.lib.chalmers.se/publication/169636

Notice: Changes introduced as a result of publishing processes such as copy-editing and formatting may not be reflected in this document. For a definitive version of this work, please refer to the published source. Please note that access to the published version might require a subscription. 


\title{
Non-invasive EEG Source Localization using Particle Swarm Optimization: A Clinical Experiment
}

\author{
Yazdan Shirvany ${ }^{1}$, Student Member, IEEE, Fredrik Edelvik ${ }^{2}$, Stefan Jakobsson ${ }^{2}$, Anders Hedström ${ }^{3}$, \\ Qaiser Mahmood ${ }^{1}$, Artur Chodorowski ${ }^{1}$ and Mikael Persson ${ }^{1}$ Member, IEEE
}

\begin{abstract}
One of the most important steps of pre-surgical diagnosis in patients with medically intractable epilepsy is to find the precise location of the epileptogenic foci. An Electroencephalography (EEG) is a non-invasive standard tool used at epilepsy surgery center for pre-surgical diagnosis. In this paper a modified particle swarm optimization (MPSO) method is applied to a real EEG data, i.e., a somatosensory evoked potentials (SEPs) measured from a healthy subject, to solve the EEG source localization problem. A high resolution $1 \mathrm{~mm}$ hexahedra finite element volume conductor model of the subject's head was generated using T1-weighted magnetic resonance imaging data. An exhaustive search pattern and the MPSO method were then applied to the peak of the averaged SEPs data. The non-invasive EEG source analysis methods localized the somatosensory cortex area where our clinical expert expected the received SEPs. The proposed inverse problem solver found the global minima with acceptable accuracy and reasonable number of iterations.
\end{abstract}

\section{INTRODUCTION}

Epilepsy is one of the most common neurologic diseases in the world and many patients with epilepsy never receive the treatment which make them seizure free. Surgical therapy has become an important therapeutic alternative for patients with medically intractable epilepsy. The combination of the neuronal and network approaches open up for a short remark or an assumption that uncontrolled network oscillations in epilepsy need a cortical focus for high frequency activity during seizures. The spikes, the epileptiform activity in the interval between the seizures, also need cortical foci [1]. Thus, source localization of epileptic activity is a tool to delineate cortical areas/volumes with abnormal neuronal activity of cells and networks. However, correct and anatomically precise localization of the epileptic focus is mandatory to decide if resection of brain tissue is possible.

An electroencephalogram (EEG) is the most important method that is used in the clinical daily routine to find the source of activities inside the brain. The procedure of the EEG source localization deals with two problems: 1) a forward problem to find the scalp potentials for the given current source(s) inside the brain, 2) an inverse problem to estimate the source(s) that fit with the given potential distribution at the scalp electrodes.

Evolutionary optimizations such as the genetic algorithm, ant colony optimization, differential evolution, evolutionary programming and particle swarm optimization (PSO), are used extensively to optimize complicated nonlinear optimizations. As the EEG source localization is a highly nonlinear

\footnotetext{
${ }^{1}$ Y. Shirvany, Q. Mahmood, A. Chodorowski and M. Persson are with the Department of Signals and Systems, Chalmers University of Technology and MedTechWest Center, Göteborg, Sweden. yazdan. shirvany at chalmers.se

${ }^{2}$ F. Edelvik and S. Jakobsson are with the Fraunhofer-Chalmers Research Centre, Chalmers Science Park, Göteborg, Sweden.

${ }^{3}$ A. Hedström is with the Sahlgrenska Academy, Göteborg, Sweden.
}

optimization problem, therefore evolutional optimization algorithms are a capable method of obtaining best solutions for this kind of applications. In a previous study by the author [2], a modified particle swarm optimization (MPSO) method was proposed for the inverse problem and applied to synthetically produced EEG data.

In this paper, the MPSO method is applied to real EEG data. Our main goal is to propose a novel technique for resolving the EEG source localization problem in the daily routine clinical application. The real EEG data that we use in the present study was recorded from somatosensory evoked potentials (SEPs) stimulation on a healthy subject.

\section{METHOD}

\section{A. Forward problem}

The characteristic frequencies of the signals in the $\mathrm{kHz}$ range and below make the capacitive and inductive effects of the tissue negligible. Therefore, the quasi-static approximation of Maxwell's equations for the potential $\Phi$ can be used. If we denote the domain of interest as $\Omega$ (with boundary $\partial \Omega$ ) and let the tissue conductivity be $\sigma$, we have Poisson's equation

$$
\nabla \cdot(\sigma \nabla \Phi)=\nabla \cdot \mathbf{j}^{s} \text { in } \Omega,
$$

subject to the conditions

$$
\begin{aligned}
\hat{\mathbf{n}} \cdot(\sigma \nabla \Phi) & =0 \text { on } \partial \Omega \\
\Phi\left(\mathbf{x}_{\mathrm{ref}}\right) & =0 .
\end{aligned}
$$

The source current $\mathbf{j}^{s}(\mathbf{x})=\delta\left(\mathbf{x}-\mathbf{x}_{0}\right) \mathbf{M}$ is modeled by a mathematical dipole at position $\mathbf{x}_{0} \in \Omega$ with the moment $\mathbf{M} \in \mathbf{R}^{3}$. The source has a singularity at $\mathbf{x}_{0}$ and is therefore difficult to model with standard finite elements. A modified subtraction method [3] is used to circumvent this problem, where the total potential is split into two parts,

$$
\Phi=\chi \Phi^{\infty}+\Phi^{\mathrm{mod}}=F^{\infty}+\Phi^{\mathrm{mod}} .
$$

$\chi$ is a smooth cut-off function which is identically 1 in a neighborhood of $\mathbf{x}_{0}$. For convenience, we have defined the function $F^{\infty}=\chi \Phi^{\infty}$.

In (4), $\Phi^{\infty}$, is the solution to (1) in an unbounded domain with constant conductivity $\sigma^{\infty}$. The solution can in this case be formed analytically [4] as

$$
\Phi^{\infty}(\mathbf{x})=\frac{1}{4 \pi \sigma^{\infty}} \frac{\left(\mathbf{x}-\mathbf{x}_{0}\right) \cdot \mathbf{M}}{\left|\mathbf{x}-\mathbf{x}_{0}\right|^{3}} .
$$

Using (4) and (1), the new formulation reads,

$$
-\nabla \cdot\left(\sigma \nabla \Phi^{\mathrm{mod}}\right)=\nabla \cdot\left(\sigma \nabla F^{\infty}\right)-\nabla \cdot \mathbf{j}^{s} \text { in } \Omega,
$$

subject to

$$
\begin{aligned}
\hat{\mathbf{n}} \cdot\left(\sigma \nabla \Phi^{\mathrm{mod}}\right) & =-\hat{\mathbf{n}} \cdot\left(\sigma \nabla F^{\infty}\right) \text { on } \partial \Omega, \\
\Phi\left(\mathbf{x}_{\mathrm{ref}}\right) & =0 .
\end{aligned}
$$


After Applying variational and FE technique to (6) for the EEG forward problem yield a linear equation system,

$$
\mathbf{u}_{\text {elec }}^{\text {mod }}=\mathbf{R} \mathbf{u}^{\text {mod }}=\mathbf{R} \mathbf{K}^{-1} \mathbf{b}=\mathbf{T b} .
$$

where $\mathbf{K} \in R^{N \times N}$ is a sparse symmetric positive definite stiffness matrix, $\mathbf{b} \in R^{N}$ the right hand side vector with $N$ being the number of FE nodes, $\mathbf{R}$ is a restriction matrix with size $N_{\text {elec }} \times N$ and the $\mathbf{T}$ is called the transfer matrix.

As shown in (4) the total potential comes from two parts so therefore the total potential at the electrodes can be written as follows

$$
\mathbf{u}_{\text {elec }}^{\text {mod }}=\left(\mathbf{T B}\left(\mathbf{x}_{0}\right)+\mathbf{F}_{\text {elec }}^{\infty}\left(\mathbf{x}_{0}\right)\right) \mathbf{M}=\mathbf{G}\left(\mathbf{x}_{0}\right) \mathbf{M} .
$$

Here $\mathbf{F}_{\text {elec }}^{\infty}\left(\mathbf{x}_{0}\right)$ is the value of the function $F^{\infty}$ for the three polarizations at all electrodes when the dipole is located at $\mathbf{x}_{0}$, and $\mathbf{G}\left(\mathbf{x}_{0}\right)$ is called the gain matrix. We use (10) in the inverse problem to find the position of the dipole.

\section{B. Inverse Problem}

In a parametric inverse method, the number of dipoles is assumed to be fixed and their locations and moments are chosen such that the potentials at the electrodes, $\mathbf{u}_{\mathrm{elec}}$, that are computed according to (10), approximate the measured potentials $\mathbf{u}_{\text {meas }}$ well according to some criteria. Here we follow the common practice and choose the parameters such that we have the best fit in least squares sense. For one dipole we get the following minimization problem

$$
J=\min _{\substack{\mathbf{x} \in \Omega_{\text {brain }} \\ \mathbf{M} \in \mathbf{R}^{\mathrm{d}}}}\left\|\mathbf{u}_{\text {meas }}-\mathbf{G}(\mathbf{x}) \mathbf{M}\right\|,
$$

where $\Omega_{\text {brain }}$ is the brain domain and $\mathrm{d}$ the dimension. Since this is a least squares problem and $\mathbf{u}_{\text {elec }}$ depends linearly on the dipole moment it is convenient to separate the parameters in (11) and solve for the dipole moment $\mathbf{M}$ first. Define, for fixed $\mathbf{x} \in \Omega_{\text {brain }}$,

$$
J(\mathbf{x})=\min _{\mathbf{M} \in \mathbf{R}^{\mathrm{d}}}\left\|\mathbf{u}_{\text {meas }}-\mathbf{G}(\mathbf{x}) \mathbf{M}\right\| .
$$

According to the normal equations for linear least squares problems, optimality is obtained for

$$
\hat{\mathbf{M}}(\mathbf{x})=\left(\mathbf{G}^{T}(\mathbf{x}) \mathbf{G}(\mathbf{x})\right)^{-1} \mathbf{G}^{T}(\mathbf{x}) \mathbf{u}_{\text {meas }} .
$$

Substituting (13) into (12) yields after some manipulation

$$
J(\mathbf{x})=\left(\mathbf{u}_{\text {meas }}^{T}\left[\mathbf{I}-\mathbf{G}(\mathbf{x})\left(\mathbf{G}^{T}(\mathbf{x}) \mathbf{G}(\mathbf{x})\right)^{-1} \mathbf{G}^{T}(\mathbf{x})\right] \mathbf{u}_{\text {meas }}\right)^{1 / 2} .
$$

Here the optimization problem is the function of the source position only, thus the complexity of the inverse problem is reduced.

\section{PARTICLE SWARM OPTIMIZATION}

\section{A. Standard Particle Swarm Optimization}

The Particle Swarm Optimization concept was first introduced by Kennedy and Eberhart [5] in 1995 based on the social system behavior such as movement of the school of birds or the flock of fishes for finding food. Each individual in the swarm is called a particle. In the original version particles move according to the following formula:

$$
\begin{cases}\mathbf{V}_{i}^{t+1} & =\mathbf{V}_{i}^{t}+c_{1} \operatorname{Rand}()\left(\mathbf{P}_{i}-\mathbf{X}_{i}^{t}\right)+c_{2} \operatorname{Rand}()\left(\mathbf{P}_{g}-\mathbf{X}_{i}^{t}\right) \\ \mathbf{X}_{i}^{t+1} & =\mathbf{X}_{i}^{t}+\mathbf{V}_{i}^{t+1}\end{cases}
$$

where $\mathbf{X}$ and $\mathbf{V}$ represent the particle position and velocity, respectively. $\mathbf{P}_{i}$ and $\mathbf{P}_{g}$ are the personal best (pbest) and

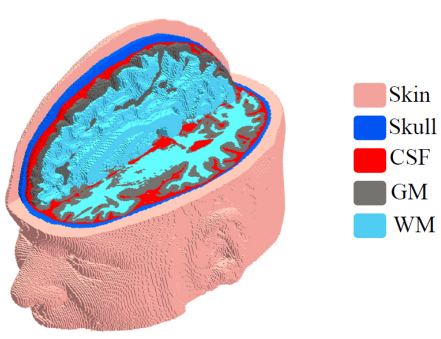

Fig. 1: The subject's FEM head model generated from the segmented MRI.

global best (gbest), respectively. $i$ represents the particle index and $t$ is time step. Rand () denotes a normally distributed one-dimensional random number with mean zero and standard deviation one. Parameters $c_{1}$ and $c_{2}$ are the cognitive and social learning rates.

\section{B. Modified Particle Swarm Optimization}

The PSO algorithm still has a risk to trap in a local minima. The pbest and gbest should not be very close to each other. If this happens the particle become inactive in certain stage of the searching steps. One way to avoid PSO to trap in local minima is mutation and using evolutionary programming (EP). In modified PSO (MPSO) we use the concept of authority [2] mixed with the mutation and EP [6] and apply them to the particle's behavior. The concept of authority means that in some steps the particles which are closer to the global best can influence the swarm performance and swarm decision more than other particles. This is because when the gbest particle is moving close to the minima, it cannot move faster than its velocity weight which is a small value during the last iterations. When PSO comes close to a minima (local or global) it can only find the global one when it has sufficiently many particles around gbest. Thus, PSO needs a lot of iterations to gather enough particles around gbest.

We extract the $R=5$ closest particles to the gbest and let them fly freely based on their memory and knowledge. This allows the PSO to have more information around gbest before lots of particles come close to it and stuck with each other. Now, the velocity update is divided into two parts as

$$
\mathbf{V}_{i}^{t+1}=w \mathbf{V}_{i}^{t}+c_{1} \operatorname{Rand}()\left(\mathbf{P}_{i}-\mathbf{X}_{i}^{t}\right)+c_{2} \operatorname{Rand}()\left(\mathbf{P}_{g}-\mathbf{X}_{i}^{t}\right),
$$

where $i=1,2, \ldots, N-R$ and

$$
\mathbf{V}_{i}^{t+1}=w \mathbf{V}_{i}^{t}+c_{1} \operatorname{Rand}()\left(\mathbf{P}_{i}-\mathbf{X}_{i}^{t}\right)
$$

where $r=N-R+1, \ldots, N$ and $w$ is the inertia weight advised by [7], [8]. The $R$ nearest particles to gbest are re-selected in each iteration to ensure that the particles which moved away from the gbest loose their authority and update their velocity according to (16). The following parameters are selected for the MPSO coefficients: $w=$ linear from 0.9 to $0.4, c_{1}=$ $0.8, c_{2}=0.4$ and swarm size $=30$, in addition the MPSO uses adaptive swarm size [9] during its searching progress. Reflecting walls are used as boundary conditions for MPSO. When a particle hits the boundary in one of the dimensions, the sign of the velocity in that dimension is changed and the particle is reflected back toward the solution space. This boundary condition keeps the particles inside the searching space. 


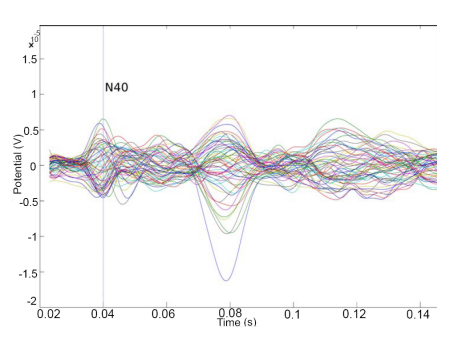

(a)

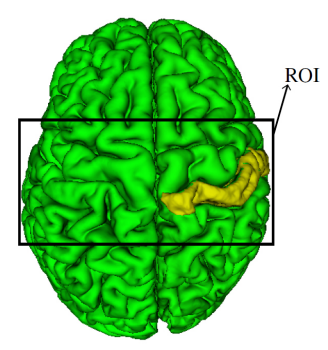

(b)
Fig. 2: a) Butterfly plot of the averaged N40 at the 57 EEG electrode positions. b) The grey matter surface (S-I view): the yellow patch indicates the right hemisphere somatosensory cortex and the box is the ROI.

\section{Anatomical Constraints}

The EEG signals are generated by currents flowing in the apical dendrites of cortical pyramidal cells [10] so the searching area could be restricted only to the cortex sheet of the brain. We use this information and add it to MPSO searching. For the anatomical constraint MPSO solely evaluates (14) for the particles which are placed in the grey matter and assign a high value to others. The MPSO starts from grey matter and in this way it ends up in the grey matter, also this constraint helps to avoid trapping the inverse problem in false local minima in other tissues. This reduces the number of evaluations and thus the computational time significantly.

\section{MRI AND EEG DATA}

\section{A. Image Segmentation}

The T1-weighted MR data of the subject's head was generated on a $1.5 \mathrm{~T}$ Philips at the Department of clinical neurophysiology, Sahlgrenska University Hospital, Göteborg, Sweden. The resolution of each voxel in the MR data is $1 \times 1 \times 1 \mathrm{~mm}$. The segmentation of the five tissues, i.e., cerebrospinal fluid (CSF), white matter, grey matter, skull and skin, was done by FSL [11]. The segmented tissues were checked and corrected manually by a clinical expert.

Here the high resolution model is necessary to accurately model the CSF compartment and the thin area of the skull. We generated the $1 \mathrm{~mm}$ hexahedra FE head model with approximately 2.8 million cells from segmented MRI, see Fig. 1. The following conductivities were then assigned to the FE compartments based on their segmentation labels and the isotropic reference model [12]: $\mathrm{skin}=0.43 \mathrm{~S} / \mathrm{m}$, skull $=$ $0.0042 \mathrm{~S} / \mathrm{m}$ (skull to skin conductivity ratio of approximately $1: 100), C S F=1.538 \mathrm{~S} / \mathrm{m}$, grey matter $=0.33 \mathrm{~S} / \mathrm{m}$, and white matter $=0.142 \mathrm{~S} / \mathrm{m}$. The $61 \mathrm{EEG}$ electrodes were placed on the subject's head based on the 10/10 EEG electrode system [13].

\section{B. Somatosensory Evoked Potential}

Evoked potentials are the electrical signals generated by the nervous system in response to sensory stimuli. Auditory, visual, and somatosensory stimuli are used commonly for clinical evoked potential studies. SEPs consist of a series of waves that reflect sequential activation of neural structures along the somatosensory pathways. In this study the left posterior tibial nerves at the subject's ankle were stimulated. The selected nerves were stimulated with monophasic square pulses, 300 microseconds in duration and the stimuli was delivered by using a constant current stimulator with 5.2

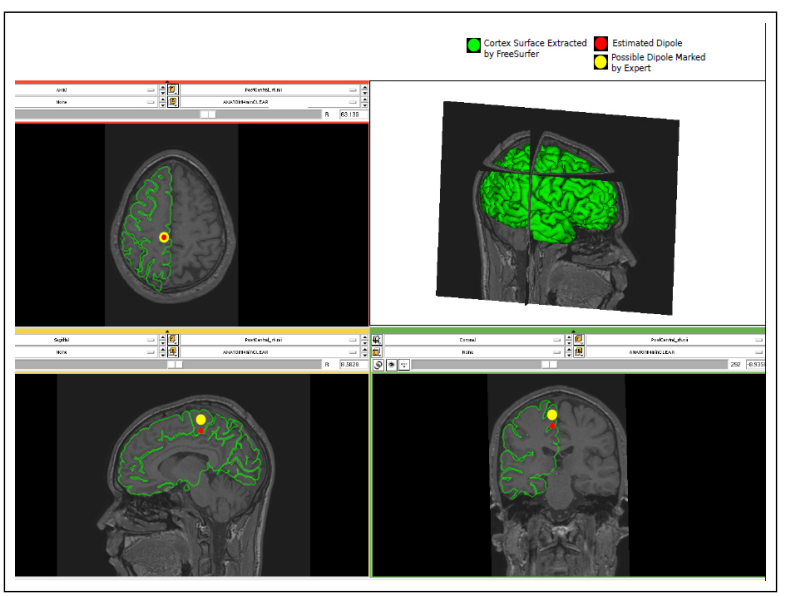

Fig. 3: The yellow area is marked by a expert as possible source position, the red area is the estimated dipole position. Note: The MRI space in Slicer is flipped compare to the anatomical space.

$m A$. To increase SNR signal averaging was used. SEPs components typically are named by their polarity and typical peak latency in the normal population. For example, N40 is a negativity that typically peaks at 40 milliseconds after the stimulus. The N40 predominantly reflects activity of neurons in the posterior tibial nerve at the primary somatosensory cortex [14] and we use this potential as input for the inverse problem. After the EEG filtering and artifact removing we found out that some of the electrodes were still noisy. We think this was because of bad connections of those electrodes with the subject's head surface during the measurement and they were removed from the data set. Four electrodes were rejected from the averaging progress, i.e., F7, Fz, CP1 and O2. Fig. 2a shows the average of 260 stimulations for N40 at the 57 EEG electrode positions.

\section{RESUlTS AND DisCUSSION}

\section{A. Validation}

Validation of the source localization is difficult, because there exist no "ground truth" to compare with. We have taken two approaches to validate our method: first we use the physiological knowledge on localization of motor and sensory functions [15] based on clinical expertise and second we do an exhaustive search pattern, i.e., inversion was performed for each possible source location in the motor and sensory cortex area inside a region of interest shown in Fig. $2 b$, and the site producing the smallest residual norm was selected as the best possible source location. The ROI has approximately 280000 grey matter voxels.

Fig. 3 shows the results of the exhaustive search plotted in the subject's head MRI. In Fig. 3 the yellow circle is the possible source position marked by a clinical expert and the red circle is the estimated source.

\section{B. MPSO results}

The 30 particles were initialized in the ROI box shown in Fig. 2b. These particles were generated randomly inside the grey matter with normal distribution. The optimization method was terminated by either of the following two criterias: 1) the minimum relative error value from the exhaustive search was obtained or 2) the maximum number of evaluations was reached. Fig. 4 shows the convergence curve for the MPSO. The MPSO found the global minima 


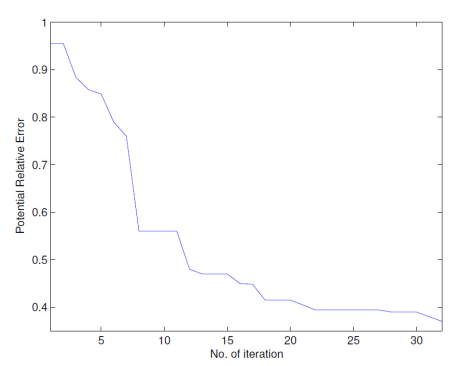

Fig. 4: The MPSO convergence curve for the cost function. The relative error between these two potentials is 0.25

after around 450 evaluations and the optimization progress terminated because of criteria 1 . We can easily see that the number of evaluations in MPSO are less then $0.15 \%$ of the total number of exhaustive search evaluations.

Fig. 5 shows the surface EEG topographic mapping for the measured EEG potential and the calculated EEG potential from the estimated source. From Fig. 5a one can see that the original source has a radial direction. Fig. 5b shows that the estimated source can reconstruct the EEG topographic mapping with a good approximation corresponding to the measured EEG. Moreover, the estimated source also has the same radial direction as the original source. We can see that the calculated and measured potentials agree well. As we can see in Fig. 3, the $\mathrm{x}$ - and y-coordinates of the estimated source are correctly located corresponding to the marked position by a clinical expert but the z-coordinate is a little bit deeper than expected [15]. There exist some error source in this experiment which need to be investigated further, e.g., electrode misplacement, EEG signal noise, segmentation uncertainties or tissue anisotropy influences in the head model.

\section{CONClusions}

In this paper, the ability of a new optimization method was tested for EEG source localization. The new method is a modified version of particle swarm optimization. In this new approach, positions and orientations of dipoles are optimized to obtain the best least squares fit with the measured EEG signals. For the forward problem, we built a realistic highresolution finite element head volume conductor based on a T1-weighted MR dataset for the construction of a five-tissue model, i.e., grey matter, white matter, CSF, skull and skin.

Somatosensory evoked potentials (SEPs) stimulation by an electrical pulse on the left posterior tibial nerve at the healthy subject's ankle, was recorded with 61 EEG electrodes placed on the scalp. Based on physiological knowledge, a somatosensory cortex generates the SEPs and its position is known a priori with a good approximation. Although this position slightly differs between individuals the variations are small [15] and we can use this knowledge to validate our results. Moreover, an exhaustive search pattern was performed for each possible source location in the motor and sensory cortex. This used for validation of the MPSO results. The EEG source localization results obtained from MPSO gave the same results as exhaustive search but with significantly lower computational complexity.

Based on our clinical expert the $\mathrm{x}$ - and $\mathrm{y}$-coordinates of the estimated source are correctly located but the $\mathrm{z}$-coordinate is a little deeper than expected. The results are promising from a clinical point of view. This deep estimation of the source maybe because of electrode misplacement noise, EEG

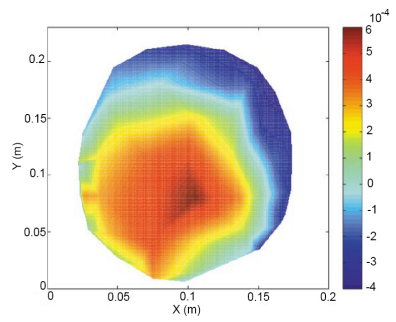

(a)

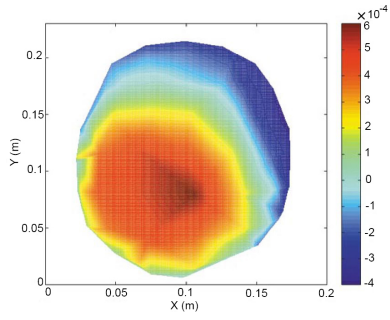

(b)
Fig. 5: Surface EEG topographic mapping: a) Measured EEG, b) Calculated EEG from the estimated source.

signal noise, segmentation uncertainties or tissue anisotropy influences in the head model. The influence of these source of errors needs to be investigated further.

\section{ACKNOWLEDGMENT}

The authors would like to thank Prof. Mikael Elam and M.Sc. Simon Bergstrand from the Sahlgrenska University Hospital for providing us the hospital measurement resources to measure the EEG and MRI data.

\section{REFERENCES}

[1] H. Laufs, "Functional imaging of seizures and epilepsy: evolution from zones to networks," Current Opinion in Neurology, vol. 25, 2012.

[2] Y. Shirvany, F. Edelvik, S. Jakobsson, A. Hedström, and M. Persson, "Application of particle swarm optimization in epileptic spike EEG source localization." Applied Soft Computing, Submitted, 2011.

[3] C. H. Wolters, H. Köstler, C. H. J. Möller, L. Grasedyck, and W. Hackbusch, "Numerical mathematics of the subraction method for the construction of a current dipole in EEG source reconstruction using finite element head models," SIAM J. Scientific Computing, vol. 30 , no. 1 , pp. $24-45,2007$

[4] C. H. Wolters, M. Kuhn, A. Anwander, and S. Reitzinger, "A parallel algebraic multigrid solver for finite element method based source localization in the human brain," Computing and Visualization in Science, vol. 5, no. 3, 2002.

[5] J. Kennedy and R. Eberhart, "Particle swarm optimization," in Neural Networks, 1995. Proceedings., IEEE International Conference on, vol. 4, 1995, pp. $1942-1948$ vol.4.

[6] K. Chellapillam, "Combining mutation operators in evalutionary programming," IEEE transaction of Evolutionary Computation, pp. 9196, 1998

[7] Y. Shi and R. Eberhart, "A modified particle swarm optimizer," in Evolutionary Computation Proceedings, 1998. IEEE World Congress on Computational Intelligence., The 1998 IEEE International Conference on, 1998, pp. $69-73$

[8] Y. Shi and R. C. Eberhart, "Parameter selection in Particle Swarm Optimization," in EP '98: Proceedings of the 7th International Conference on Evolutionary Programming VII. London, UK: SpringerVerlag, 1998, pp. 591-600.

[9] C. M., "Think locally, act locally: The way of life of cheap-pso, an adaptive pso." 2001.

[10] S. Murakami and Y. Okada, "Contributions of principal neocortical neurons to magnetoencephalography and electroencephalography signals," The Journal of Physiology, vol. 575, no. 3, pp. 925-936, 2006.

[11] M. W. Woolrich, S. Jbabdi, B. Patenaude, M. Chappell, S. Makni, T. Behrens, C. Beckmann, M. Jenkinson, and S. M. Smith, "Bayesian analysis of neuroimaging data in FSL," NeuroImage, vol. 45, pp. S173 - S186, 2009

[12] S. Gabriel, R. Lau, and C. Gabriel, "The dielectric properties of biological tissues: III parametric models for the dielectric spectrum of tissues," Phys Med Biol, vol. 41, no. 11, pp. 2271 - 2293, 1996.

[13] V. Jurcak, D. Tsuzuki, and I. Dan, "10/20, 10/10, and 10/5 systems revisited: Their validity as relative head-surface-based positioning systems," NeuroImage, vol. 34, no. 4, pp. 1600 - 1611, 2007.

[14] M. J. Aminoff, Electrodiagnosis in Clinical Neurology. Churchill Livingstone, Elsevier, 2005.

[15] W. Penfield and E. Boldrey, "Somatic motor and sensory representation in the cerebral cortex of man as studied by electrical stimulation," Brain, vol. 60, no. 4, pp. 389-443, 1937. 